Urol. Prax. 2021 $23: 76-83$

https://doi.org/10.1007/s41973-021-00141-2

Angenommen: 6. Mai 2021

Online publiziert: 9 . Juni 2021

○ Der/die Autor(en) 2021

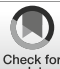

Ralf Anding ${ }^{1} \cdot$ Stefan Müller ${ }^{2} \cdot$ Helge Seifert ${ }^{1}$

' Urologische Klinik, Universitätsspital Basel, Basel, Schweiz

${ }^{2}$ Urologische Klinik, Universitätsklinikum Bonn, Bonn, Deutschland

\title{
Therapiemöglichkeiten der weiblichen Belastungsinkontinenz
}

\section{Einleitung}

Die Jahrtausendwende brachte in Bezug auf das Thema weibliche Inkontinenz viele wesentliche Änderungen mit sich, die auch die aktuelle Therapie gut 20 Jahre später noch bestimmen. Im deutschsprachigen Raum wurde der Begriff der Stressinkontinenz von der Bezeichnung Belastungsinkontinenz abgelöst, um klarzustellen, dass es sich um ein physisches, nicht um ein psychisches Problem handelt. Nach einer Änderung der ICS-Definition wurde zudem jeder unwillkürliche Urinverlust als Inkontinenz bezeichnet, nicht nur, wenn dieser ein soziales oder hygienisches Problem darstellte.

Mehrere gross angelegte nationale und europäische Studien erfassten die tatsächliche Prävalenz der verschiedenen Inkontinenzformen und rückten somit dieses Tabuthema nicht nur im medizinischen Bereich ins rechte Licht, sondern trugen es auch in die breite Öffentlichkeit. So entpuppte sich die Inkontinenz als echtes Volksleiden, mit einer höheren Prävalenz als Hypertonie, Diabetes oder Depression. Jede 3. Frau ist in ihrem Leben von Belastungsinkontinenz betroffen, insbesondere in der Menopause, und ca. 55\%, wenn noch Drangsymptome hinzugerechnet werden (gemischte Inkontinenz).

Das anatomische und pathophysiologische Verständnis der weiblichen Inkontinenz wurde in dieser Zeit um die Integraltheorie (www.integraltheory.org; PE Papa Petros) von Petros u. Ulmsten [1] sowie das Hammock-Modell von DeLancey [2] erweitert und bis heute entscheidend geprägt. Daraus resultierte ebenfalls eine Revolution der Therapie, nämlich die Einführung des TVT durch Ulmsten et al. [3], welches sich durch hohe Effektivität und geringe Invasivität in kurzer Zeit weltumspannend als Therapiestandard durchsetzte. Mit der Verwendung von Polypropylen wurde ein Material gefunden, welches die zuvor oft problembehaftete Verwendung alloplastischer Materialien biokompatibler und anwenderfreundlicher machte und die Operationszahlen der weiblichen Inkontinenz insgesamt in die Höhe trieb. Ebenfalls erhöhte sich die Anzahl der Anwender in Urologie und Gynäkologie drastisch. Zahllose Modifikationen der Bänder und eine Erweiterung des Einsatzes in den Bereich der Senkungsoperationen führten jedoch zu einer Häufung von Komplikationen, was zunächst in den USA zu zahlreichen Prozessen und zu drastischen Einschränkungen der Zulassungen durch die FDA führte. In der Folge wurde der Einsatz alloplastischer Bänder und Netze in vielen Ländern (z.B. Australien, Neuseeland, Grossbritannien) gänzlich verboten.

In diesen Ländern steht heute die optimale Versorgung von Patientinnen mit Belastungsinkontinenz in Frage, da die aktuelle Generation von Operateuren die klassischen Behandlungsmethoden ohne synthetisches Material oft nicht mehr erlernt hat und somit Behandlungsalternativen fehlen.

\section{Anmerkungen zur Diagnostik}

Die Belastungsinkontinenz der Frau ist eine klinische Diagnose, wegweisend sind eine gezielte Anamnese und die klinische Untersuchung. Zunächst muss der genaue Anlass des Urinverlustes eruiert werden, typischerweise eine plötzliche abdominale Druckerhöhung, z. B. beim Husten, Niesen, Lachen, Springen, Bücken, Heben. Ein Harndrang besteht zuvor nicht, jedoch ist der Urinverlust bei stärker gefüllter Blase naturgemäss grösser. Die Symptomatik sollte mit standardisierten Fragebögen und einem Trink- und Miktionsprotokoll genauer erfasst werden. Die klinische Einteilung des Schweregrades der Belastungsinkontinenz erfolgt klassischerweise nach Stamey:

- Grad 1: Urinverlust bei plötzlichem Anstieg des abdominalen Drucks, z. B. Husten, Niesen, Lachen, aber niemals nachts im Bett

- Grad 2: Urinverlust bei geringerer Belastung, z. B. Gehen, Aufstehen von einem Stuhl oder aus dem Bett

- Grad 3: Urinverlust ohne Beziehung zur Belastung oder Körperposition, auch im Liegen

Der tatsächliche Urinverlust wird durch die Anzahl der verbrauchten Vorlagen nur unzureichend wiedergegeben, da sehr unterschiedliche Größen verwendet werden. Aus diesem Grund bewährte sich das Wiegen der Vorlagen, sodass der tatsächliche Flüssigkeitsverlust objektiv erfasst werden kann. Die Mengen werden dann innerhalb eines 24-h-Zeitraums addiert. Dies spiegelt die Alltagssituation besser wider als ein kurzzeitiger Belastungstest, z. B. der sog. 1-h-Pad-Test (Vorlagentest) nach Klarskov u. Hald [4] oder der 20-min-Test nach Hahn u. Fall [5]. Dennoch können diese Tests, insbe- 

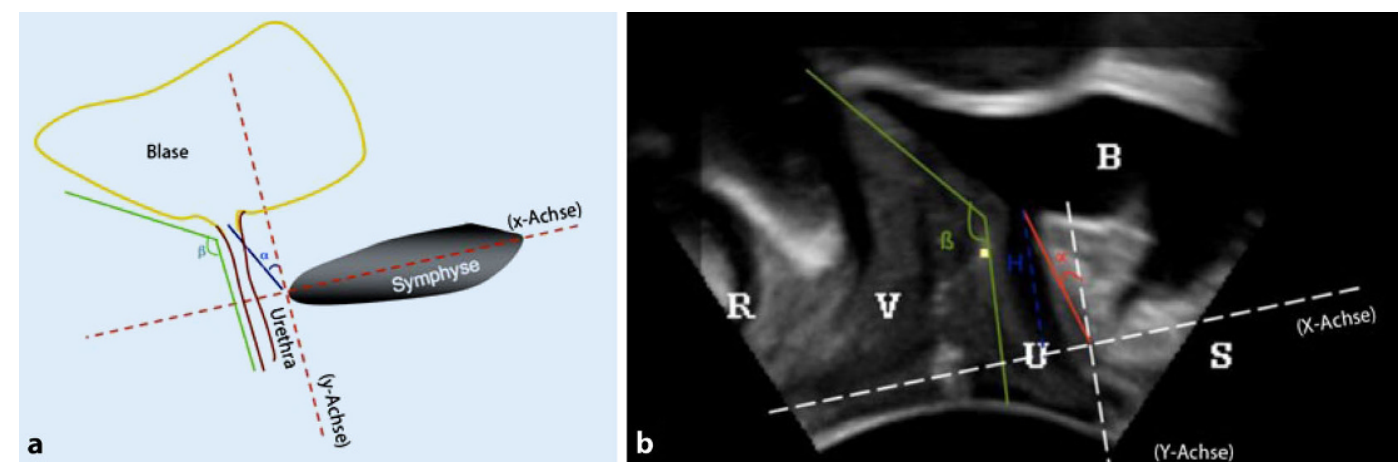

Abb. $1 \Delta$ a Schemadarstellung der Beckenbodenkoordinaten bei der Frau, b Anwendung des Koordinatensystems auf ein UItraschallbild bei der Frau, $R$ Rektum, $V$ Vagina, UUrethra, BBlase, SSymphyse. (Nachdruck mit Genehmigung aus:[6] $\odot$ SpringerVerlag Berlin Heidelberg 2015. Diese Abbildung fällt nicht unter die Creative Commons CC BY-Lizenz dieser Publikation)

sondere zur Therapiekontrolle, sinnvoll sein.

Weiterhin müssen Erkrankungen und Medikamente, Operationen, insbesondere im Beckenbereich, Zahl und Art von Geburten, Risikofaktoren (Menopause [lokaler Östrogenmangel], Rauchen [häufiges Husten], Adipositas [Beckenbodenüberlastung]) und die alltägliche (berufliche und private) körperliche Belastung abgefragt werden. Dazu bewährten sich umfassende Fragebögen, die die Patientin schon im Wartebereich ausfüllen kann.

Die Basisdiagnostik beinhaltet eine Urinuntersuchung, eine Sonographie mit Restharnbestimmung und eine vaginale Untersuchung in Steinschnittlage. Der Untersuchungsgang sollte aus praktischer Erwägung in umgekehrter Reihenfolge durchgeführt werden, damit die vaginale Einstellung mit einer möglichst gefüllten Blase erfolgen kann. Dabei sollten unbedingt Spekula mit 2 einzelnen Blättern nach Kristeller benutzt werden und keinesfalls die aus der Vorsorgeuntersuchung bekannten Spekula nach Cusco. Für eine suffiziente Beurteilung des Beckenbodens und der Harnröhre müssen alle 3 Kompartimente (vorderes, mittleres, hinteres) separat untersucht werden. Besonderes Augenmerk gilt dem vorderen Kompartiment mit der Harnröhre. Dabei wird die Patientin zum kräftigen Husten aufgefordert (Vorsicht Untersucher: in etwa $10 \mathrm{~cm}$ Abstand die Hand vorhalten!) und die Exkursion der Harnröhre und der Urinverlust beobachtet. Bei positivem Hustentest (sichtbarer Urinverlust) wird das Manöver mit beidseitiger vaginaler Anhebung der Harnröhre wiederholt, entweder mit einem Instrument (z.B. Kornzange) oder 2 Fingern (MarshallBonney-Test). Dies beschreibt zum einen die mögliche Ursache der Inkontinenz (mangelnde Beckenbodenunterstützung der Harnröhre oder hypermobile Harnröhre [Typ-I-/-II-Inkontinenz nach $\mathrm{Mc}$ Guire]), zum anderen ist bei positivem M-B-Test (kein Urinverlust bei lateraler Anhebung der Harnröhre) schon der Weg zur Therapie (zumeist in Form eines spannungsfreien Bandes) gebahnt. Ein negativer M-B-Test (Urinverlust trotz lateraler Anhebung) zeigt eine Typ-IIIInkontinenz (hypotone/starre Harnröhre) an und bedarf einer spezialisierten Behandlung. Bei negativem Hustentest kann dieser auch im Stehen wiederholt werden, wobei die Patientin mit etwas geöffneten Beinen auf eine Unterlage gestellt wird und zum Husten und Wippen auf den Fussballen aufgefordert wird.

Ein weiteres Instrument zur Befundobjektivierung ist die perineale, vaginale oder Introitussonographie, die besonders bei Komorbiditäten im Beckenboden (Senkung, Voroperationen, Bestrahlung) in jedem Fall eingesetzt werden sollte (-Abb. 1). Die Beurteilung der Anatomie, Lagebeziehungen und Beweglichkeit der Beckenorgane setzt eine entsprechende Schulung voraus. Eine weitere (radiologische) Bildgebung erübrigt sich damit in der Regel und bleibt speziellen Fällen vorbehalten [6].

Eine Indikation zur urodynamischen Untersuchung ist gegeben, wenn die Anamnese und/oder Basisuntersuchung einen unklaren Befund ergeben, eine gemischte oder nicht eindeutige Inkontinenz vorliegt, eine neurologische Erkrankung mit vermuteter Blasenfunktionsstörung vorliegt oder in anderen speziellen Fällen, wenn dies die Therapieentscheidung beeinflusst. Bei klinisch eindeutiger Belastungsinkontinenz ohne Komorbiditäten hat eine urodynamische Untersuchung keinen Einfluss auf das Behandlungsergebnis und ist daher nicht indiziert. Zudem ist die Untersuchung stark situations- und untersucherabhängig und somit nur bedingt objektiv.

\section{Konservative Therapie}

Wie bei vielen anderen Erkrankungen ist eine Ausschaltung von Risikofaktoren wünschenswert, aber oft nicht realistisch. In der amerikanischen PRIDEStudie („program to reduce incontinence by diet and exercise") reduzierte sich die Anzahl der Inkontinenzepisoden bei übergewichtigen Patientinnen nach 6 Monaten um 58\%, 27\% der Teilnehmerinnen konnten allein durch Gewichtsreduktion von ihrer Belastungsinkontinenz kuriert werden [7]. Bei extrem übergewichtigen Patientinnen (BMI $\varnothing 46,9 \mathrm{~kg} / \mathrm{m}^{2}$ ) konnte durch bariatrische Chirurgie die Prävalenz der Belastungsinkontinenz nach $1 \mathrm{Jahr}$ von $42,5 \%(39,2-45,8 \%)$ auf $9,8 \%(8,3-11,7 \%)$ gesenkt werden. Eine entsprechende Beratung und Unterstützung übergewichtiger Patientinnen werden unbedingt empfohlen [8].

Grundsätzlich steht die konservative Therapie der Belastungsinkontinenz im- 
mer an erster Stelle. Hierdurch wird ein Bewusstsein für den eigenen Beckenboden geschaffen, mit gezielter Aktivierung und Relaxation des urethralen Sphinkters und Mobilisierung muskulärer Reserven. Dies kann nur eine Physiotherapie durch besonders geschulte Beckenbodentherapeuten leisten. Als Ergänzung können Biofeedbackmethoden oder eine Elektrostimulation mit entsprechenden Geräten eingesetzt werden. Eine Cochrane-Analyse mit 31 Studien und 1874 Frauen zeigte eine 8-mal höhere Wahrscheinlichkeit der Heilung in der aktiven Beckenbodentherapiegruppe gegenüber der Kontrollgruppe [9]. Der erwünschte kontinenzverbessernde Effekt kann jedoch nur im aktiven Übungszeitraum aufrechterhalten werden, es muss der Patientin daher die Notwendigkeit fortwährender Übungen verdeutlicht werden. Viele Patientinnen ziehen daher einen kleinen Eingriff mit hoher Heilungswahrscheinlichkeit vor (s. Abschnitt Alloplastische Bänder).

Auch die medikamentöse Behandlung mit Duloxetin kann die Belastungsinkontinenz nur im Behandlungszeitraum um ca. $50 \%$ reduzieren und eignet sich somit eher für die milde Inkontinenz. Als Serotoninwiederaufnahmehemmer werden die postsynaptische Rezeptoraktivierung des $\mathrm{N}$. pudendus in seinem spinalen Kern (Nucleus Onuf) gesteigert und konsekutiv die Kontraktilität des externen Sphinkters verbessert. Wichtig bei der Behandlung mit Duloxetin sind die einschleichende Dosierung und auch das langsame Ausschleichen der Therapie, ein wichtiger limitierender Faktor ist nämlich die Verträglichkeit der Behandlung.

Vaginale Pessare stellen zumeist eine temporäre Therapie dar und werden von 70-90\% der Frauen toleriert. Insbesondere Ringpessare mit einer Verstärkung unterhalb der Harnröhre (Urethrapessare) können die Belastungsinkontinenz signifikant verbessern [10]. Entscheidend sind eine gute Auswahl, Anpassung und Kontrolle der Pessartherapie, um eine Schädigung der Vaginalhaut zu verhindern. Insbesondere bei älteren Patientinnen sollte die Behandlung mit einer lokalen Östrogenisierung (Östriol bzw. Estriol) kombiniert werden, wel-
Urol. Prax. 2021 · 23:76-83 https://doi.org/10.1007/s41973-021-00141-2

(c) Der/die Autor(en) 2021

\section{R. Anding $\cdot$ S. Müller $\cdot$ H. Seifert}

\section{Therapiemöglichkeiten der weiblichen Belastungsinkontinenz}

\section{Zusammenfassung}

Die Jahrtausendwende ging in Bezug auf das Thema weibliche Inkontinenz mit vielen wesentlichen Änderungen einher, der Begriff Stressinkontinenz wurde von Belastungsinkontinenz abgelöst, die Integraltheorie von Petros u. Ulmsten sowie das Hammock-Modell von DeLancey erweiterten das Verständnis der weiblichen Inkontinenz, das TVT (Tensionfree vaginal tape) setzte sich in kurzer Zeit weltumspannend als Therapiestandard durch. Die vielversprechend gestartete Stammzelltherapie wird trotz inzwischen vorliegender klinischer Studien kontrovers beurteilt. Etablierte Therapieverfahren sind zunächst die konservative Therapie mit gezieltem Beckenbodentraining, minimalinvasive Verfahren wie die suburethrale Injektion, klassische Operationsverfahren wie die Faszienzügelplastik und die Kolpo- suspension mit ihren Modifikationen sowie die alloplastischen suburethralen Bänder (TVT, TOT [Transobturator tape], SIS [Single incision sling]), entweder spannungsfrei oder adjustierbar. Die Operationsmethoden liefern auch im Langzeitverlauf insgesamt gute Ergebnisse bei geringerer Morbidität der Bandverfahren. In Anbetracht der weltweiten Zulassungsbeschränkungen alloplastischer Bänder sollten die urogynäkologische Expertise in klassischen Operationstechniken aufrechterhalten werden und neue Entwicklungen den IDEAL (Innovation, Development, Exploration, Assessment, Long-term study)Empfehlungen folgen.

Schlüsselwörter Konservativ · Indikation · Faszie · Klassisch Bänder

\section{Options thérapeutiques lors d'incontinence d'effort chez la femme}

\section{Résumé}

Le tournant du millénaire a apporté de nombreuses modifications importantes dans le domaine de l'incontinence féminine; la notion d'incontinence de stress a été remplacée par celle d'incontinence d'effort, la théorie intégrale de Petros et d'Ulmsten ainsi que la théorie du hamac de DeLancey ont élargi la compréhension de l'incontinence féminine et la TVT s'est rapidement établie dans le monde entier en tant que traitement standard. Le traitement par cellules souches, initialement prometteur, fait encore l'objet de controverses malgré les études cliniques désormais disponibles. Les traitements établis comprennent le traitement non chirurgical par des exercices ciblés du plancher pelvien, les procédés mini-invasifs tels que l'injection sous-urétrale et les procédés chirurgicaux classiques tels que la plastie de soutènement par bande de fascia et la colposuspension avec leurs modifications ainsi que les bandelettes alloplastiques sous-urétrales (TVT, TOT, SIS) sans tension ou ajustables. Les méthodes chirurgicales atteignent aussi à long terme des résultats globaux satisfaisants, avec une moindre morbidité que les méthodes à bandelettes. Face aux restrictions concernant l'homologation des bandelettes alloplastiques à travers le monde, l'expertise urogynécologique dans les techniques chirurgicales classiques doit être maintenue et les nouveaux développements doivent se conformer aux recommandations de I'IDEAL.

\section{Mots clés}

Conservateur · Indication · Fascia · Classique . Bandelettes che ebenfalls einen wichtigen Faktor der medikamentösen Kontinenztherapie darstellt.

Die eigentlich nicht angestrebte Hilfsmittelversorgung rückt in einer immer älter werdenden Bevölkerung wieder mehr in den Vordergrund. Hierbei kommen aufsaugende (Einlagen) und ableitende (Katheter) Hilfsmittel zur Anwendung. Insbesondere in Pflege- einrichtungen beträgt die Prävalenz der Inkontinenz ca. $70 \%$, ca. $25 \%$ der Pflegezeit wird für die Inkontinenzversorgung aufgewendet. Zudem verursachen die verbrauchten Hilfsmittel hohe volkswirtschaftliche Kosten, die kontinuierlich ansteigen. Auch die Entsorgung dieser kontaminierten Einmalmaterialien nimmt einen immer höheren Stellenwert ein. Bei beiden For- 


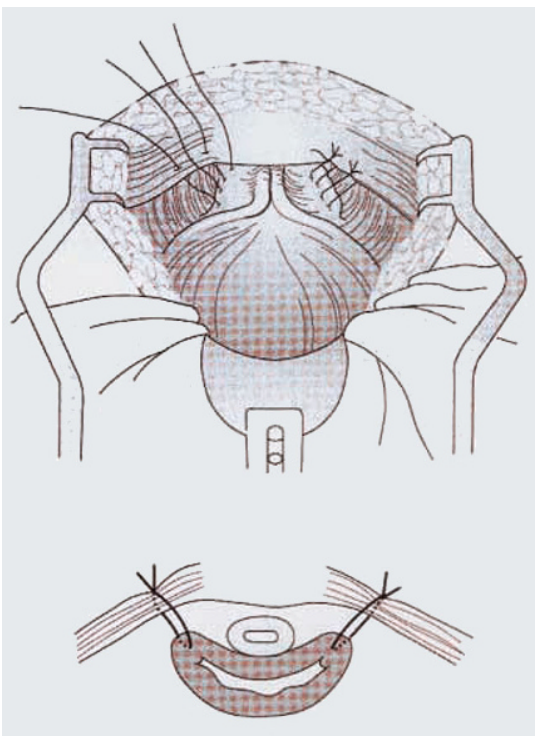

Abb. $2 \Delta$ Kolposuspension nach Burch. Die nichtresorbierbaren Fäden werden locker mit respektvollem Abstand zur Harnröhre zwischen vorderer Vaginalwand und Cooper-Ligament gelegt. (Nachdruck mit Genehmigung aus: [32] CSpringer-Verlag 2001. Diese Abbildung fällt nicht unter die Creative Commons CC BY-Lizenz dieser Publikation)

men der Hilfsmittelversorgung stehen hygienische Aspekte ganz besonders im Vordergrund. Durch gute Lagerung, regelmässige Wechsel von Einlagen, Kathetern, Beuteln und Hautpflege sollen Infektionen und Druckulzera vermieden, zumindest aber rechtzeitig erkannt und behandelt werden. $\mathrm{Zu}$ bedenken ist auch, dass durch die häufig gebrauchte sog. Dauerableitung der Blase deren Harnspeicherfähigkeit irreversibel verloren geht und sich dadurch die Situation bei vielen Patientinnen verschlechtert.

\section{Minimal-invasive Therapie}

Zur Erhöhung des Blasenauslasswiderstands kann die Harnröhre künstlich verengt werden. Dies geschieht durch urethroskopische suburethrale Injektion verschiedener Substanzen an 2-4 Punkten distal des Blasenhalses, sodass eine möglichst konzentrische Einengung erzeugt wird und das Urothel in der Mitte des Harnröhrenlumens Kontakt hat (Koaptation). Wenn sich das Lumen im Spülstrahl des Zystoskops gerade nicht mehr öffnet, sollte die Injektion zur Vermeidung einer Überkorrektur beendet werden. Dies kann sich technisch

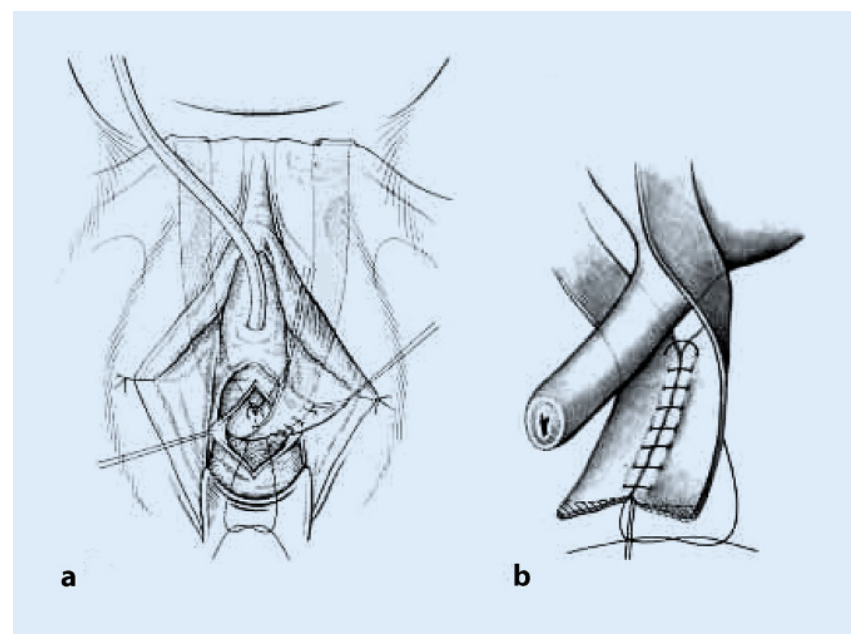

Abb. 3 A Klassische Faszienzügelplastik nach Narik u. Palmrich. a retropubisch nach unten gezogene Streifen aus der Rektusfaszie, b Vereinigung unter der Harnröhre mit "Kletternaht". (Nachdruck mit Genehmigung aus: [33] @Springer-Verlag 2001. Diese Abbildung fällt nicht unter die Creative Commons CC BY-Lizenz dieser Publikation)

bei der Frau durchaus anspruchsvoll gestalten und verlangt einige Übung. Früher kam biologisches Material zur Anwendung, wie autologes Fett oder heterologes Kollagen, welches aber recht rasch wieder abgebaut wurde und heutzutage keine Rolle mehr spielt. Aktuell werden synthetische Materialien (Silikon [Macroplastique ${ }^{\circledR}$ ], Dextranomer/ Hyaluronsäure [Deflux ${ }^{\circledR}$ ], Kalziumhydroxylapatit [Coaptite ${ }^{\circledR}$ ], PolyacrylatPolyalkohol-Kopolymer [PPC; Opsys ${ }^{\circledR}$, Vantris $^{\circledR}$ ], Polyakrylamid [Bulkamid ${ }^{\circledR}$ ]) verwendet, die sich als biokompatibel erwiesen haben. Der Vorteil liegt in der schnellen und wenig belastenden Prozedur, die in Lokalanästhesie durchgeführt und auch leicht wiederholt werden kann. Der Nachteil liegt in der eingeschränkten Effektivität und Wirkdauer der Behandlung, zudem kann sich durch mehrfache Injektionen in der Folge eine periurethrale Fibrose entwickeln und die Inkontinenz sogar verschlechtern. Die Verwendung dieser sog. „bulking agents" kommt somit insbesondere für ältere Patientinnen in Frage, für die eine Operation bzw. eine Narkose ein besonderes Risiko bedeuten würden.

Die ebenfalls zur Jahrtausendwende vielversprechend gestartete Stammzelltherapie befindet sich weiterhin im experimentellen Stadium und wird trotz inzwischen vorliegender klinischer Studien (352 Patientinnen in 9 Studien) kontro- vers beurteilt. Hierbei wird Muskelgewebe z.B. aus dem M. deltoideus entnommen (MDC, ,muscle-derived cells"), einige Zeit kultiviert und als Suspension in den urethralen Sphinkter injiziert. Die Injektionstechnik entspricht hierbei im Wesentlichen den „bulking agents“. Die meisten Patientinnen profitierten von der Behandlung mit vollständiger Kontinenz in bis $\mathrm{zu} 79 \%$, allerdings zumeist nur über kurze Zeiträume [11]. Die Kostensituation und die rechtlichen Vorgaben in vielen Ländern schränken die Methode weiterhin ein.

\section{Klassische Operationsverfahren}

Die Technik der Inkontinenzoperationen mit Suspension des Blasenhalses bzw. der proximalen Harnröhre wurde schon vor über 100 Jahren entwickelt [12] und im Grundsatz bis heute nur unwesentlich verändert. Es werden in der klassischen Technik nach Narik u. Palmrich 2 Streifen aus der vorderen Rektusfaszie präpariert und von dort aus wie ein Zügel nach unten um den Blasenhals oder die Harnröhre geschlungen (• Abb. 2). Unterhalb werden die Faszienstreifen dann durch Nähte wieder vereinigt, der Grad der Suspension kann hierbei individuell angepasst werden [13]. Die Methode wurde dann v. a. durch McGuire modifiziert, der die longitudinalen Faszienstreifen durch einen freien transversalen Streifen ersetz- 

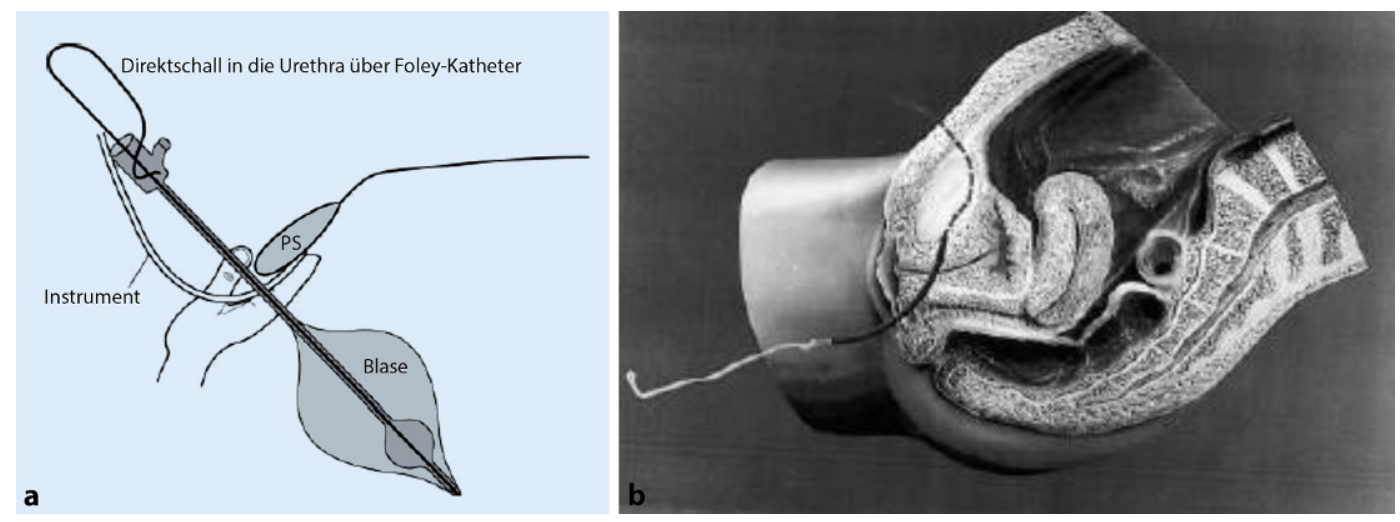

Abb. $4 \varangle$ Schema (a) und Anatomiemodell (b) der TVT-Einlage von U. Ulmsten. PS Ramus superior ossis pubis. (Nachdruck mit Genehmigung aus: [34] OSpringer-Verlag 2001. Diese Abbildung fällt nicht unter die Creative Commons CC BY-Lizenz dieser Publikation)

te, welcher weiterhin wie ein Zügel um die proximale Harnröhre gelegt wurde, aber durch Nähte nach oben gehalten und hier über der Rektusfaszie fixiert wurde („sling on a string“; [14]). Heute spielt diese modifizierte Faszienzügelplastik (pubovaginale Schlingenplastik) nur noch eine untergeordnete Rolle, sollte aber insbesondere in Rezidivfällen eine Therapieoption bleiben.

Die originale Kolposuspension nach Burch beinhaltete eine $\mathrm{zu}$ nah an der Harnröhre gelegte und zu straffe Nahtfixierung am Cooper-Ligament [15], sodass Blasenentleerungsstörungen und Drangbeschwerden oft die Folge waren. Die Fixierung erfolgt mit nichtresorbierbaren, vorzugsweise weichen, multifilen Nähten (z.B. Ti-Cron ${ }^{\text {Tw }}$ ), eine evtl. notwendige Korrektur kann daher nur mittels Lösung der Fäden („Burch-takedown manoeuvre") erfolgen. Es wurden verschiedene Modifikationen, z. B. nach Tanagho [16] oder Cowan u. Morgan [17] präsentiert, die Operation, welche über Jahrzehnte als sog. Goldstandard der weiblichen Inkontinenztherapie galt, blieb aber mit dem Namen Burch verbunden (• Abb. 3). Die Ergebnisse haben den Test der Zeit bestanden, mit einer Gesamtkontinenzrate von $69-88 \%$ im Langzeitverlauf bis über 10 Jahre [18]. Um die Jahrtausendwende wurden erste Serien von laparoskopisch durchgeführten Kolposuspensionen publiziert, die etwas schlechtere Ergebnisse als die offene OP zeigten, zum einen aus methodischen Gründen, da häufig nur eine Naht je Seite gelegt wurde (normalerweise 2-3 Nähte je Seite), wahrscheinlich aber auch durch die fehlende taktile Kontrolle des Operateurs, die gerade bei dieser
OP sehr wichtig ist [19]. Das Verfahren sollte weiterhin zum festen Armamentarium der Urogynäkologie gehören, insbesondere bei jungen Patientinnen.

\section{Alloplastische Bänder}

Die Idee, defektes Gewebe durch ein Material „mit der Dichte und Festigkeit einer Faszie oder Sehne“ zu ersetzen, hatte schon Theodor Billroth: „das Geheimnis der radikalen Heilung der Hernie wäre gelöst" sprach er $1878 \mathrm{zu}$ seinem Schüler Vincenz Czerny. Bis zur Realisierung dieses Traums vergingen noch über 100 Jahre, denn nicht die Festigkeit implantierbarer Kunststoffe war das grösste Problem, sondern die Biokompatibilität. Es wurde daher bei der Herstellung von geflochtenen Band- und Netzstrukturen auf bewährtes Nahtmaterial zurückgegriffen. Erst mit der Einführung des TVT („tension free vaginal tape“) durch Ulmsten et al. [3] verbreitete sich ein günstiges und leicht zu verarbeitendes Material (Polypropylen), dessen Eigenschaften (Typ-1-Mesh nach Amid: monofilamentär, makroporös, leichtgewichtig, weich) den bis dahin verwendeten überlegen war. Aktuell wird das Polypropylen bei genauerer struktureller, mikrobiologischer und immunologischer Betrachtung als Standardmaterial der Beckenbodenchirurgie wieder kontrovers gesehen [20].

Die neue retropubische TVT-Technik (• Abb.4) folgte der zuvor postulierten Integraltheorie, sodass keine aktive Suspension von Harnröhre/Blasenhals in kranialer Richtung erfolgte, sondern eine passive kaudale Unterstützung der Harnröhre. Diese erfolgte auch nicht mehr proximal oder gar am Blasenhals, sondern in der Mitte der Harnröhre als Ersatz der defekten pubourethralen Ligamente und Korrektur einer dadurch hypermobilen Harnröhre (Typ-II-Inkontinenz nach McGuire). Das spannungsfreie Band entfaltet seine Wirkung erst durch eine abdominale Druckerhöhung (Husten, Niesen usw.), wobei es dem kranialen Druck und der hierbei ausgelösten Rotation der proximalen Harnröhre einen unbeweglichen Widerstand entgegensetzt. Die Harnröhre kann sich durch diese temporäre Obstruktion nicht mehr öffnen, und die Patientin bleibt kontinent [21]. Es gibt zahllose Studien zum TVT und späterer artverwandter Produkte mit dem Nachweis hoher Effektivität, die interessanteste ist sicherlich die fortgesetzte Nachbeobachtung des Originalkollektivs von 90 Patientinnen aus Uppsala, Stockholm und Helsinki, welche auch nach 17 Jahren (11 Patientinnen und Ulf Ulmsten waren inzwischen verstorben) noch eine Heilungsrate von 87,2\% angibt [22]. Die subjektive Zufriedenheit mit dem Ergebnis betrug in diesem Kollektiv zuvor 95,3\% nach 5, 97,6\% nach 7 und 97,0\% nach 11 Jahren.

Auch die 2001 von Delorme [23] etablierte transobturatorische Technik (TOT [„transobturator tape“]) zeigte nach anfänglichen, materialbedingten Problemen (Uratape ${ }^{\circledR}$, später ObTape ${ }^{\circledR}$, s. Abschnitt Anmerkungen zu den FDAWarnungen) in vielen Langzeituntersuchungen und Metaanalysen gleich gute Heilungsergebnisse, sodass TVT und TOT inzwischen häufig als MUS („midurethral slings") zusammengefasst werden. Unterschiede bestehen in den Risiken, wobei die retropubische 

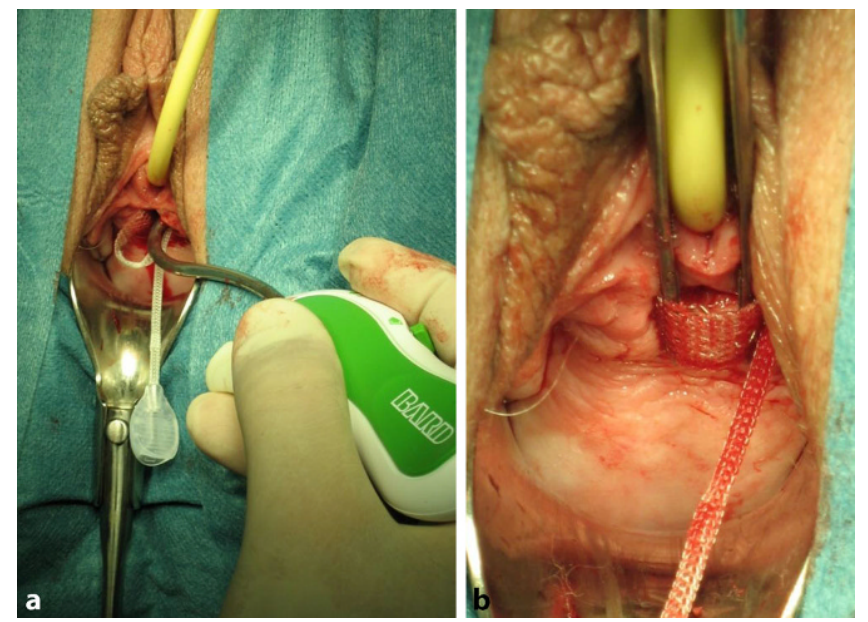

Abb. $5<$ a Implantation eines adjustierbaren SIS (hier AJUST ${ }^{T M}$, BARD, inzwischen nicht mehr erhältlich) mit einer einzigen kleinen vaginalen Inzision, b Adjustierung der Bandspannung unterhalb der Harnröhrenmitte

Technik des TVT häufiger zu Blasenverletzungen (2,5-11,7\%), Hämatomen (0,5-2,5\%), Blasenentleerungsstörungen $(2,8-38 \%)$ und Infekten $(0,4-31,5 \%)$ führt, die transobturatorische Technik des TOT häufiger mit Vaginalperforationen sowie Beschwerden und Infektionen im Adduktorenbereich verbunden ist. In 28 randomisierten Studien mit 15.855 Patientinnen zeigte sich eine signifikant höhere subjektive (OR: 0,59, $p=0,0003$ ) und objektive (OR: 0,51, $p=0,001)$ Kontinenzrate nach MUS als nach Burch-Kolposuspension [24]. Patientinnen nach MUS und PVS hatten ähnliche Kontinenzraten, bei einer höheren operationsbedingten Morbidität nach PVS [25].

Eine noch weniger invasive Methode stellen die sog. "mini slings“ oder SIS („single incision sling“) dar, welche nur einen kleinen vaginalen Zugang benötigen und in der Membrana obturatoria mit kleinen Widerhaken verankert werden (•Abb. 5). Auch hierbei fand eine Evolution der Implantate statt, inzwischen sind die hohen Kontinenzraten denen der MUS ebenbürtig, auch in der Langzeitbeobachtung, bei geringerer Morbidität und weniger Komplikationen [26, 27]. Auch nach Voroperationen, bei Rezidivinkontinenz oder bei narbigstarrer Harnröhre (Typ-III-Inkontinenz nach McGuire) bieten diese adjustierbaren Bandsysteme Vorteile, da die spannungsfreien MUS in dieser Situation weniger wirksam sind, als bei Typ-I/-II-Inkontinenz [28]. Die Anlage einer adäquaten Bandspannung ist in dieser Situation der Schlüsselfaktor des Erfolgs
[29]. Ebenso erwies sich das SIS wegen seiner geringen Invasivität bei Adipositas, De-novo-Belastungsinkontinenz nach Zystozelenkorrektur und bei älteren Patientinnen (Lokalanästhesie!) als besonders geeignet.

\section{Anmerkungen zu den FDA- Warnungen}

In der Vergangenheit konnten sowohl in den USA als auch in Europa Medizinprodukte ohne klinische Tests am Patienten zugelassen werden, indem sie sich auf die Ähnlichkeit zu einem bereits zugelassenen Produkt bezogen. Die ProteGen ${ }^{\circledR}$-Schlinge von Boston Scientific's wurde bereits 1999 wieder vom Markt genommen und war wohl „nie in eine menschliche Vagina vor der Zulassung implantiert worden" schrieb der Gynäkologe L. Lewis Wall aus St. Louis, USA, Mitautor einer Petition an die FDA zum Verbot aller nichtresorbierbaren Mesh-Implantate [30]. Dennoch entwickelten und vermarkteten viele weitere Firmen ihre Bänder auf der Grundlage des ProteGen ${ }^{\circledR}$-Designs. Hierzu gehörte auch das ObTape ${ }^{\circledR}$ der Firma Mentor Porgès, welches 2006 wegen Komplikationen von der Firma selbst wieder vom Markt genommen wurde (auch in unserem Patientengut machten wir damit ebensolche unerfreulichen Erfahrungen). Trotzdem überschwemmte die Industrie den Markt mit immer neuen Produkten. Unter dem Blickwinkel des „marketing“ lohnte sich dieses Engagement auch, denn weltweit steigt die Zahl älter werdender, übergewichti- ger Patientinnen mit Harninkontinenz. Möglicherweise lockten auch rasch steigende Fallzahlen in einer immer stärker kommerzialisierten Medizin viele Kollegen, diese Operationen anzubieten, obwohl sie sich vorher kaum mit der Thematik befasst hatten. In einer Umfrage unter $10 \%$ aller amerikanischen Urologen, die sich um die Board-Rezertifizierung bemühten (in den USA alle 10 Jahre nötig!), gaben $34 \%$ an, Beckenbodenoperationen durchzuführen, aber nur 3\% hatten ein Fellowship in Urogynäkologie absolviert. Auch in Deutschland, wo im Gegensatz zu der Schweiz keine Schwerpunktbezeichnung Urologie der Frau oder Urogynäkologie existiert, kann jeder Facharzt für Urologie oder Gynäkologie solche Operationen durchführen, obwohl seine operativen Fähigkeiten niemals objektiviert bzw. geprüft wurden.

Mit zunehmender Verwendung deralloplastischen Materialien stieg die Zahl der Komplikationen und Revisionsoperationen. Bereits im Herbst 2008 veröffentlichte die FDA eine erste Warnung, nachdem seit 2005> 1000 Mesh-assoziierte Komplikationsfälle von 9 verschiedenen Herstellern gemeldet worden waren. Zumeist handelte es sich um Erosionen, Infektionen, Schmerzen und Miktionsprobleme, aber auch einzelne schwerwiegende Fälle wie Gefäß- und Darmverletzungen. In der Konsequenz wurden nur ein spezielles Training für diese Operationen sowie eine umfassende Aufklärung der Patienten empfohlen. In der Praxisänderte sich daher zunächst nichts. Im Juli 2011 veröffentlichte die FDA ein Update ihrer Warnung, da 2874 weitere Komplikationen bis Ende 2010 gemeldet worden waren, die man nun nicht mehr als seltene Ereignisse betrachtete. Die FDA verpflichtet seit Februar 2011 Hersteller von Netzimplantaten zu retrospektiven klinischen Studien ihrer Produkte. Dies führte dazu, dass mehrere Hersteller ihre Produkte vom Markt zurückzogen.

Dennoch ist das allogene Material bei korrekter Indikationsstellung und korrekter Operationsweise sehr hilfreich, auch unter Inkaufnahme erhöhter Risiken und materialbedingter Komplikationen. Die IDEAL-Empfehlungen (I: ,in- 
novation“; D: „development“; E: „exploration“; A: „assessment“; L: „longterm study") wären ein Instrument [31], um neue Operationsmethoden zu beschreiben, weiterzuentwickeln, Ergebnisse über längere Zeiträume objektiv zu erfassen und somit die Grundlage einer besseren Patientenversorgung zu schaffen, gleichzeitig aber auch die sinnvolle Weiterentwicklung der verwendeten Materialien zu ermöglichen.

\section{Fazit für die Praxis}

- Die Belastungsinkontinenz der Frau ist eine klinische Diagnose von hoher Prävalenz.

- Zur Objektivierung können eine gezielte Anamnese, Fragebögen, Miktionsprotokolle, Vorlagenverbrauch und Pad-Tests eingesetzt werden.

- Risikofaktoren (z. B. Menopause, Rauchen, Adipositas) sollten erkannt und die Patientinnen entsprechend beraten werden.

- Die Basisdiagnostik beinhaltet Urinuntersuchung, Sonographie und vaginale Untersuchung.

- Die konservative Therapie (Physiotherapie, Duloxetin, Pessare, Hilfsmittel) kann in der Praxis eingeleitet werden.

- Die operative Therapie ist bei korrekter Indikationsstellung langfristig effektiv. Für Patientinnen mit Risikofaktoren stehen auch minimalinvasive Verfahren zur Verfügung.

\section{Korrespondenzadresse}

PD Dr. Ralf Anding
Urologische Klinik,
Universitätsspital Basel
Spitalstraße 21, 4031 Basel,
Schweiz
ralf.anding@usb.ch

Funding. Open access funding provided by University of Basel

\section{Einhaltung ethischer Richtlinien}

Interessenkonflikt. R. Anding, S. Müller und H. Seifert geben an, dass kein Interessenkonflikt besteht.
Für diesen Beitrag wurden von den Autoren keine Studien an Menschen oder Tieren durchgeführt. Für die aufgeführten Studien gelten die jeweils dort angegebenen ethischen Richtlinien.

pen Access. Dieser Artikel wird unter der Creative Commons Namensnennung 4.0 International Lizenz veröffentlicht, welche die Nutzung, Vervielfältigung Bearbeitung, Verbreitung und Wiedergabe in jeglichem Medium und Format erlaubt, sofern Sie den/die ursprünglichen Autor(en) und die Quelle ordnungsgemäß nennen, einen Link zur Creative Commons Lizenz beifügen und angeben, ob Änderungen vorgenommen wurden.

Die in diesem Artikel enthaltenen Bilder und sonstiges Drittmaterial unterliegen ebenfalls der genannten Creative Commons Lizenz, sofern sich aus der Abbildungslegende nichts anderes ergibt. Sofern das betreffende Material nicht unter der genannten Creative Commons Lizenz steht und die betreffende Handlung nicht nach gesetzlichen Vorschriften erlaubt ist, ist für die oben aufgeführten Weiterverwendungen des Materials die Einwilligung des jeweiligen Rechteinhabers einzuholen.

Weitere Details zur Lizenz entnehmen Sie bitte der izenzinformation auf http://creativecommons.org/ licenses/by/4.0/deed.de.

\section{Literatur}

1. Petros PE, Ulmsten UI (1990) An integral theory of female urinary incontinence. Experimental and clinical considerations. Acta Obstet Gynecol Scand Suppl 153:7-31. https://doi.org/10.1111/j.16000412.1990.tb08027.x

2. DeLancey JO (1994) Structural support of the urethra as it relates to stress urinary incontinence: the hammock hypothesis. Am J Obstet Gynecol 170(6):1713-1720; discussion 1720-1723. https:// doi.org/10.1016/s0002-9378(94)70346-9

3. Ulmsten $U$, Henriksson $L$, Johnson $P$, Varhos $G$ (1996) An ambulatory surgical procedure under local anesthesia for treatment of female urinary incontinence. Int Urogynecol J Pelvic Floor Dysfunct 7:81-86. https://doi.org/10.1007/BF01902378

4. Klarskov P, Hald T (1984) Reproducibility and reliability of urinary incontinence assessment with a 60 minute test. Scand J Urol Nephrol 18(4):293-298. https://doi.org/10.1016/S00029343(97)89520-4

5. Hahn I, Fall M (1991) Objective quantification of stress urinary incontinence: a short, reproducible, provocative pad-test. Neurourol Urodyn 10(5):475-481

6. Soljanik I, Brocker K, Solyanik O, Stief CG, Anding R, Kirschner-Hermanns R (2015) Bildgebung bei Harninkontinenz. Urologe 54:963-971. https:// doi.org/10.1007/s00120-015-3872-6

7. Subak LL, Wing R, West DS, Franklin F, Vittinghoff $\mathrm{E}$, Creasman JM, Richter HE, Myers D, Burgio KL, Gorin AA, Macer J, Kusek JW, Grady D; PRIDE Investigators (2009) Weight loss to treat urinary incontinence in overweight and obese women N Engl J Med 360(5):481-490. https://doi.org/10. 1056/NEJMoa0806375

8. Marcelissen T, Anding R, Averbeck M, HannaMitchell A, Rahnama'i S, Cardozo L (2019) Exploring the relation between obesity and urinary incontinence: Pathophysiology, clinical implications, and the effect of weight reduction
ICI-RS2018. Neurourol Urodyn 38Suppl5:S18-S24. https://doi.org/10.1002/nau.24072

9. Dumoulin C, Cacciari LP, Hay-Smith EJC (2018) Pelvic floor muscle training versus no treatment or inactive control treatments, for urinary incontinence in women. Cochrane Database Syst Rev. https://doi.org/10.1002/14651858.CD005654. pub4

0. Wu Y, Welk B (2019) Revisiting current treatment options for stress urinary incontinence and pelvic organ prolapse: a contemporary literature review. Res RepUrol 11:179-188. https://doi.org/10.2147/ RRU.S191555

11. Barakat B,FrankeK, Schakaki S, HijaziS, Hasselhof $\mathrm{V}$ Vögeli TA (2020) Stem cell applications in regenerative medicine for stress urinary incontinence: a review of effectiveness based on clinical trials. Arab J Urol 18(3):194-205. https://doi.org/10.1080/ 2090598X.2020.1750864

12. Goebell R (1910) Zur operativen Beseitigung der angeborenen Incontinentia Vesicae. Dtsch Gynaekol Urol 2:187-191

13. Narik G, Palmrich AH (1962) A simplified sling operation suitable for routine use. Am J Obste Gynecol 84:400-405. https://doi.org/10.1016/ 0002-9378(62)90139-4

14. Mcguire EJ, Lytton B (1978) Pubovaginal sling procedure for stress incontinence. JUrol 119(1):82-84 https://doi.org/10.1016/s0022-5347(17)57390-5

15. Burch JC (1961) Urethrovaginal fixation to Cooper's ligament for correction of stress incontinence, cystocele, and prolapse. Am J Obstet Gynecol 81:281-290. https://doi.org/10.1016/s00029378(16)36367-0

16. Tanagho EA (1976) Colpocystourethropexy: the way we do it. J Urol 116:751-753. https://doi.org/ 10.1016/s0022-5347(17)58997-1

17. Cowan W, Morgan HR (1979) A simplified retropubicurethropexyin the treatment of primary and recurrent urinary stress incontinence in the female. Am J Obstet Gynecol 133(3):295-298. https://doi.org/10.1016/0002-9378(79)90682-3

18. Lapitan MCM, Cody JD, Mashayekhi A (2017) Open retropubic colposuspension for urinary incontinence in women. Cochrane Database Syst Rev. https://doi.org/10.1002/14651858. CD002912.pub7

19. Veit-Rubin N, Dubuisson J, Ford A, Dubuisson JB, Mourad S, Digesu A (2019) Burch colposuspension. Neurourol Urodyn 38:553-562. https://doi.org/10. 1002/nau.23905

20. Rovner E, de Tayrac R, Kirschner-Hermanns R, Veit-Rubin N, Anding R (2020) Is polypropylene mesh material fundamentally safe for use as a reconstructive material in vaginal surgery: ICIRS 2019? Neurourol Urodyn 39 Suppl 3:S132-S139. https://doi.org/10.1002/nau.24312

21. Dietz HP, Wilson PD, Gillies K, Vancaillie TG (2000) How does the TVT achieve continence? Neurourol Urodyn 19:393-394

22. Nilsson CG, Palva K, Aarnio R, Morcos E, Falconer C (2013) Seventeen years' follow-up of the tensionfree vaginal tape procedure for female stress urinaryincontinence. Int Urogynecol J 24:1265-1269. https://doi.org/10.1007/s00192-013-2090-2

23. Delorme E (2001) Transobturator urethral suspension: mini-invasive procedure in the treatment of stress urinary incontinence in women. Prog Urol 11(6):1306-1313

24. Fusco F, Abdel-Fattah M, Chapple CR, Creta M, La Falce S, Waltregny D, Novara G (2017) Updated systematic review and meta-analysis of the comparative data on colposuspensions, pubovaginal slings, and midurethral tapes in 
the surgical treatment of female stress urinary incontinence. Eur Urol 72(4):567-591. https://doi. org/10.1016/j.eururo.2017.04.026

25. Trabuco EC, Klingele CJ, Weaver AL, McGree ME, Lightner DJ, Gebhart JB (2009) Medium-term comparison of continence rates after rectus fascia or midurethral sling placement. Am J Obstet Gynecol 200(3):300.e1-300.e6.https://doi.org/10 1016/j.ajog.2008.10.017

26. Zhang $P$, Fan $B$, Zhang $P$, Han $H, X u$ Y, Wang B Zhang $X$ (2015) Meta-analysis of female stress urinary incontinence treatments with adjustable single-incision mini-slings and transobturator tension-free vaginal tape surgeries. BMC Urol 15:64.https://doi.org/10.1186/s12894-015-0060 3

27. Manso M, Botelho F, Silva C, Cruz F (2021) Minislings: do they stand the test of time? A 10-year cohort. Urol Int 105:143-147. https://doi.org/10. 1159/000511648

28. Rezapour M, Falconer C, Ulmsten U (2001) Tension-free vaginal tape (TVT) in stress incontinent women with intrinsic sphincter deficiency (ISD) - a long-term follow-up. Int Urogynecol J 12 Suppl 2:S12-S14. https://doi.org/10.1007/ s001920170005

29. Anding R, Schoen M, Kirschner-Hermanns R, Fisang C, Müller SC, Latz S (2017) Minimally invasive treatment of female stress urinary incontinence with the adjustable single-incision sling system (AJUST ${ }^{\mathrm{TM}}$ ) in an elderly and overweight population. Int Braz J Urol 43(2):280-288. https:// doi.org/10.1590/S1677-5538.IBJU.2015.0751

30. Wall LL, Brown D (2010) The perils of commercially driven surgical innovation. Am J Obstet Gynecol 202(1):30.e1-4. https://doi.org/10.1016/j.ajog. 2009.05.031

31. Gerullis $H$, Klosterhalfen B, Borós M, Lammers B, Eimer C, Georgas E, Otto T (2013) IDEAL in meshes for prolapse, urinary incontinence, and hernia repair. Surg Innov 20(5):502-508. https://doi.org/ 10.1177/1553350612472987

32. Petri E (2001) Die Kolposuspension zur Behandlung der weiblichen Stressinkontinenz. Urologe A 40:292-299. https://doi.org/10.1007/ s001200170039

33. Hampel C, Hohenfellner M, Melchior S, Thüroff JW (2001) Schlingenplastiken in der Therapie der weiblichen Harninkontinenz. Urologe A 40:274-280. https://doi.org/10.1007/ s001200170036

34. Ulmsten U (2001) The basic understanding and clinical results of tension-free vaginal tape for stress urinary incontinence. Urologe A 40:269-273. https://doi.org/10.1007/s001200170035

Hinweis des Verlags. Der Verlag bleibt in Hinblick auf geografische Zuordnungen und Gebietsbezeichnungen in veröffentlichten Karten und Institutsadressen neutral.

\section{Sven Perner, Verena-Wilbeth Sailer \\ Histopathologische Diagnostik der Prostatastanzbiopsie}

Heidelberg: Springer-Verlag GmbH Deutschland, 2020, 201 S., 220 Abb., (ISBN: $978-$ 3-662-60642-1), 89,99 EUR

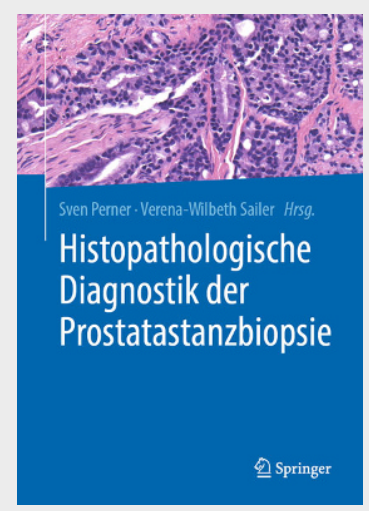

In 2020 erschien im Springer-Verlag das erste deutschsprachige Werk zur histopathologischen Diagnostik der Prostatastanzbiopsie. Sowohl die Herausgeber Sven Perner und Verena Sailer als auch die zahlreichen Mitautoren sind anerkannte Experten auf dem Gebiet des Prostatakarzinoms.

Gerade kleine Foci fraglich atypischer Drüsen sind angesichts der Zunahme von Prostatastanzbiopsaten und deren bildegebungsgesteuerten zielgenauen Entnahme im Einsendegut häufig eine Herausforderung im diagnostischen Alltag. Sowohl hierzu als auch zum breiten differentialdiagnostischen Spektrum benigner Läsionen geben die Herausgeber und die zahlreichen namhaften Autoren aus dem deutschsprachigen Raum praxisnahe Erläuterungen und Empfehlungen.

Es wird zudem ausführlich auf die aktuellen Empfehlungen zur Befunderstellung eingegangen, immer mit einem besonderen Augenmerk auf die klinischen Bedürfnisse der Urologie. Ergänzt wird das Buch um Kapitel zur Klinik, zur bildgebenden Diagnostik sowie zur Molekularpathologie des Prostatakarzinoms

Hervorgegangen ist dieses praxisorientierte Lehrbuch, dass seinen festen Platz neben dem Mikroskop finden wird, aus dem mehrfach durchgeführten IAP-Seminar zur Prostatapathologie, das die Herausgeber erstmals im November 2018 gegeben haben. Dieses Buch bietet sowohl dem Einsteiger als auch dem erfahrenen Diagnostiker eine fundierte Wissensvermittlung und stärkt die zentrale Rolle, die die histopathologische Diagnostik der Prostatastanzbiopsie als Grundlage jeder Risikostratifizierung und Therapieplanung einnimmt.

\section{Prof. Dr. Martin Anlauf}

Institut für Pathologie, Zytologie und Molekularpathologie in

Wetzlar, Limburg, Wettenberg und Bad Hersfeld

Anlauf@patho-uegp.de 